\title{
On the parametric characterization of the transversal spatial structure of laser pulses
}

\author{
C. Martínez, F. Encinas-Sanz, J. Serna, P.M. Mejías, R. Martínez-Herrero \\ Departamento de Óptica, Facultad de Ciencias Fisicas, Universidad Complutense, 28040 Madrid, Spain
}

Received 5 November 1996; revised 10 February 1997; accepted 6 March 1997

\begin{abstract}
From the application of a simple analytical model to describe a laser pulse, the transversal spatial structure of the pulse amplitude is linked to its (measurable) second-order intensity moments, integrated along the pulse duration. These formulae are applied to some examples. The experimental values correspond to TEA $\mathrm{CO}_{2}$ laser pulses, for which the dependence of the beam quality on the presence of nitrogen in the gas mixture and also on the size of an intracavity diaphragm has also been investigated.
\end{abstract}

\section{Introduction}

As is well known, on the basis of the so-called intensity-moment formalism, a number of parameters have been introduced in the literature to characterize the spatial profile of cw laser beams [1-5]. In addition, it has recently been shown [6] that, under quite general suplementary conditions, such parameters (integrated throughout the pulse duration) propagate according to the usual $A B C D$ laws. The key parameters commonly handled are beam width and beam quality (along with the position of the beam waist), which provide a global information about the spatial characteristics of the field. The aim of the present work is to get further insight about the spatial structure of the pulsed beam in terms of the above measurable parameters.

Thus, in the next section the overall intensity moments are defined for pulsed beams. In Section 3, from the application of a simple (scalar) analytical model to describe a laser pulse, a number of equations are derived which relate the transversal spatial structure of the beam to the usual second-order intensity moments. These formulae are applied in Section 4 to some illustrative examples. The selected values of the characteristic parameters we use in the examples correspond to TEA $\mathrm{CO}_{2}$ laser pulses, with the presence of an adjustable aperture inside the laser resonator for mode selection, and with (or without) nitro- gen in the gas mixture. Finally, the main conclusions are summarized in Section 5.

\section{Definitions}

Let us assume a light beam whose amplitude at plane $z=0$ is denoted by the function $g(x, t)$ (for simplicity, we will consider in this paper the bidimensional case). We can introduce the Fourier transforms

$\psi(x, \omega)=\int g(x, t) \exp (-i \omega t) \mathrm{d} t$,

$G(u, \omega)=\int \psi(x, \omega) \exp (-i k u x) \mathrm{d} x$,

where $k=\omega / c, x$ is the spatial variable transversal to the direction of propagation $z, u$ represents an angle of propagation (without taking the evanescent waves into account), and $\omega$ is the spectral frequency. It follows from Eqs. (1) and (2) that the squared moduli of $\psi$ and $G$ are related, respectively, to the intensity at $x$ and to the radiant intensity along the direction $u$, associated to the spectral component $\omega$. The overall second-order intensity moments can consequently be written in the usual way, i.e., the squared beam width takes the form

$\left\langle x^{2}\right\rangle=\frac{1}{\psi_{0}} \int x^{2}|\psi(x, \omega)|^{2} \mathrm{~d} x \mathrm{~d} \omega$, 
the squared far-field divergence is given by

$\left\langle u^{2}\right\rangle=\frac{1}{G_{0}} \int u^{2}|G(u, \omega)|^{2} \mathrm{~d} u \mathrm{~d} \omega$,

and the crossed parameter $\langle x u\rangle$ (related to the position of the waist plane) is determined by the formula

$$
\langle x u\rangle=\frac{c}{2 i \omega_{0} \psi_{0}} \int x\left[\left(\psi^{*}\right)^{\prime} \psi-\psi^{*} \psi^{\prime}\right] \mathrm{d} x \mathrm{~d} \omega,
$$

where $\psi_{0}=\int|\psi(x, \omega)|^{2} \mathrm{~d} x \mathrm{~d} \omega, G_{0}=\int|G(u, \omega)|^{2} \mathrm{~d} u \mathrm{~d} \omega$, the prime indicates derivation with respect to $x, c$ denotes the speed of light, and $\omega_{0}$ is the mean frequency. It is understood that the pulsed beam is averaged over the pulse transit time across the plane $z=0$. To write Eqs. (3)-(5) we are assuming, for simplicity, that $\langle x\rangle=\langle u\rangle=0$. We will also consider in the following that the fields we analyse are spectrally well-centered and locally quasimonochromatic (SCLQ) beams [6], for which the overall spatial second-order moments follow the $A B C D$ law (within the paraxial approach). For example, light pulses generated by TEA $\mathrm{CO}_{2}$ lasers belong to this type of fields.

Finally, the beam quality parameter, $Q$, can be introduced as follows:

$Q=\left\langle x^{2}\right\rangle\left\langle u^{2}\right\rangle-\langle x u\rangle^{2}$,

where $\langle x u\rangle=0$ at the waist plane. For SCLQ beams it can be shown that $[G]$

$\left\langle x^{2}\right\rangle\left\langle u^{2}\right\rangle \geq \frac{c^{2}}{4 \omega_{0}^{2}}$,

where $\left\langle x^{2}\right\rangle$ and $\left\langle u^{2}\right\rangle$ are defined by Eqs. (3) and (4) and the equality is reached by beams whose transversal profile is Gaussian. The above inequality is identical to the one that applies to $\mathrm{cw}$ laser beams.

\section{Theory}

Let us now consider a pulsed beam at its waist plane. We can write each spectral component, $\psi(x, \omega)$, in the form

$\psi(x, \omega)=\sum_{n=0}^{N} \alpha_{n} f_{n}(x, \omega)$,

where $f_{n}, n=0, \ldots, N$ represent the laser modes. Here we associate the term "modes" to definite spatial structures of the laser field, which resonate within the loaded cavity. Consequently, each value of index $n$ refers to a particular spatial structure. Note that the spatial structure will be essentially the same for any axial mode frequency within the linewidth of the laser oscillator. The existence of different $f_{n}$ would then announce the presence of different transversal modes. Also note that, since different modes involve different sets of transverse mode resonance frequencies, we can consider that

$\int f_{n}^{*}(x, \omega) f_{m}(x, \omega) \mathrm{d} \omega=0, \quad n \neq m$.

Let us now introduce the Hermite-Gauss (H-G) functions, $\varphi_{p}(x)$, which are assumed to correspond to the empty-cavity modes, i.e.,

$\varphi_{p}(x)=\beta_{p} H_{p}(\gamma x) \exp \left(-\gamma^{2} x^{2} / 2\right)$,

where $\beta_{p}$ is a constant, $H_{p}$ denotes the Hermite polynomial and $\gamma$ is a constant which is inferred from the geometry of the cavity, namely [7],

$\gamma^{-1}=\left(\frac{L}{k}\right)^{1 / 2}\left[\frac{g_{1} g_{2}\left(1-g_{1} g_{2}\right)}{\left(g_{1}+g_{2}-2 g_{1} g_{2}\right)^{2}}\right]^{1 / 4}$,

where $L$ is the length of the laser resonator, and $g_{1}=1-$ $L / R_{1}$ and $g_{2}=1-L / R_{2}$ are the resonator $g$ parameters, $R_{1}$ and $R_{2}$ being the mirror curvatures. It seems reasonable to write the pulse amplitude, $\psi(x, \omega)$, of the loaded resonator in terms of the empty-cavity modes as follows:

$\psi(x, \omega)=\gamma^{1 / 2} \sum_{p} B_{p}(\omega) \varphi_{p}(x)$,

where

$$
\begin{aligned}
B_{p}(\omega) & =\gamma^{-1 / 2} \int \psi(x, \omega) \varphi_{p}(x) \mathrm{d} x \\
& =\sum_{n=0}^{N} \alpha_{n} \int f_{n}(x, \omega) \varphi_{p}(x) \mathrm{d} x .
\end{aligned}
$$

By taking into account the recurrence properties of the H-G functions an their derivatives, we find in a completely similar way to that used in Ref. [8] the following relations:

$$
\begin{aligned}
2 \gamma^{2}\left\langle x^{2}\right\rangle= & \sum_{p}(2 p+1) R_{p p} \\
& +2 \sum_{p}[(p+2)(p+1)]^{1 / 2} \operatorname{Re}\left(R_{p, p+2}\right),
\end{aligned}
$$

$$
\begin{aligned}
4 k^{2} Q= & {\left[\sum_{p}(2 p+1) R_{p p}\right]^{2} } \\
& -4\left|\sum_{p}[(p+2)(p+1)]^{1 / 2} R_{p, p+2}\right|^{2}, \\
k\langle x u\rangle= & \sum_{p}[(p+2)(p+1)]^{1 / 2} \operatorname{Im}\left(R_{p, p+2}\right),
\end{aligned}
$$

with

$\sum_{p} R_{p p}=1$, 
where

$R_{p q}=\frac{c_{p q}}{P}=\frac{1}{P} \int B_{p}^{*}(\omega) B_{q}(\omega) \mathrm{d} \omega$

and

$P=\sum_{p} c_{p p}$

is proportional to the total energy per pulse. Note that (see Eq. (18))

$\left|R_{p q}\right|^{2} \leq R_{p p} R_{q q}$.

But $\langle x u\rangle=0$ at the waist plane, so that we have from Eq. (16)

$\sum_{p}[(p+2)(p+1)]^{1 / 2} \operatorname{Im}\left(R_{p, p+2}\right)=0$,

and Eq. (15) becomes

$$
\begin{aligned}
4 k^{2} Q= & {\left[\sum_{p}(2 p+1) R_{p p}\right]^{2} } \\
& -4\left\{\sum_{p}[(p+2)(p+1)]^{1 / 2} \operatorname{Re}\left(R_{p, p+2}\right)\right\}^{2},
\end{aligned}
$$

Let us now introduce two parameters, namely,

$$
\begin{aligned}
& s \equiv 2 \gamma^{2}\left\langle x^{2}\right\rangle, \\
& r \equiv 2 \sum_{p}[(p+2)(p+1)]^{1 / 2} \operatorname{Re}\left(R_{p, p+2}\right) .
\end{aligned}
$$

From Eqs. (14), (22)-(24) it follows that

$$
s-r=\sum_{p}(2 p+1) R_{p p},
$$

and

$$
r=\frac{s^{2}-4 k^{2} Q}{2 s} .
$$

Since $\left\langle x^{2}\right\rangle$ and $Q$ can be directly obtained from the experiment, parameters $s$ and $r$ should be considered as measurable quantities. In summary, we have that any pulsed beam (as given by Eq. (8)) must fulfil at the waist plane the following four equations (along with expression (20)):

$$
\begin{aligned}
& s-r=\sum_{p}(2 p+1) R_{p p}, \\
& r / 2=\sum_{p}[(p+2)(p+1)]^{1 / 2} \operatorname{Re}\left(R_{p, p+2}\right), \\
& 0=\sum_{p}[(p+2)(p+1)]^{1 / 2} \operatorname{Im}\left(R_{p, p+2}\right), \\
& 1=\sum_{p} R_{p p} .
\end{aligned}
$$

By means of the above equations, the transversal spatial structure of the pulsed beam is linked to the second-order intensity moments. As will become clear in the next section, coefficients $R_{p p}$ provide information about the relative contribution (weight) of each (squared) $\mathrm{H}-\mathrm{G}$ function in the expansion (12). This is why Eq. (27) might be considered, in a sense, as the main result of this section. In addition, Eq. (29) is a consequence of the fact that the beam is evaluated at the waist plane. Eq. (30) shows the normalization condition. $\Lambda \mathrm{n}$ interesting property can be derived from Eq. (28): When $r$ differs from zero, the expansion (12) should contain at least two H-G functions, which differ by two orders. The usefulness of this property will be apparent in the next section.

Note finally that the left-hand side of Eqs. (27) and (28) can be obtained from the experimental data. However, the above four equations (27)-(30) are not enough to explicitly determine all the coefficients $R_{\rho q}$. This constitutes an inherent limitation of the model regarding the possibility of inferring the detailed spatial structure of the pulsed beam from the measurement of the parameters $\left\langle x^{2}\right\rangle$ and $Q$. Nevertheless, additional experimental data and supplementary hypothesis about the behaviour of the modes can overcome this difficulty (for example, in certain practical cases, the contribution of the higher-order modes can be assumed small enough to be neglected).

\section{Application to some examples}

To illustrate what kind of information can be inferred from Eqs. (27) - (30), we will substitute on these formulae the values of the parameters $s$ and $r$ measured (along the $x$-direction) at the output of a TEA $\mathrm{CO}_{2}$ laser device. The experimental set-up is shown in Fig. 1. The ensemble pyroelectric camera (Spiricon PYROCAM I) with laser beam analyzer (Spiricon LBA-100A) provides the (squared) beam width (integrated along the complete pulse duration) at different planes after a lens (focal length $500 \mathrm{~mm}$ ). Pulse energies range from $1 \mathbf{J}$ (without nitrogen in the gas mixture) to $8 \mathrm{~J}$ (for the following gas mixture $\mathrm{CO}_{2}: \mathrm{N}_{2}: \mathrm{He}$

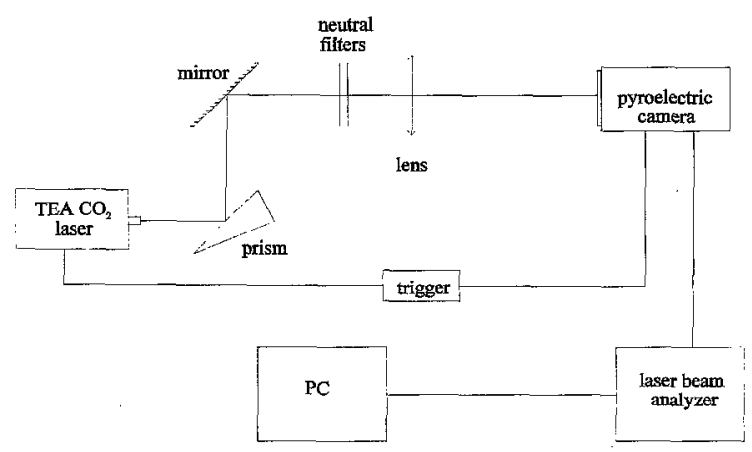

Fig. 1. Experimental set-up used to measure parameters $s$ and $r$. 


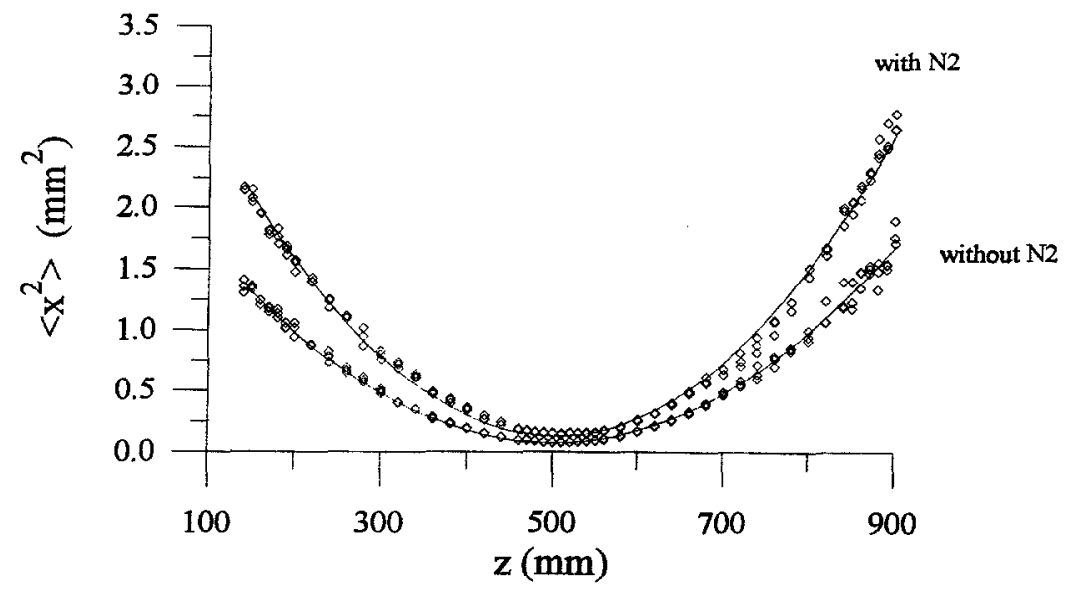

Fig. 2. Squared beam width $\left\langle x^{2}\right\rangle$ at different planes after the auxiliary lens (see Fig. 1) for $d=10 \mathrm{~mm}$. Solid lines are parabolas. Rhombs represent the experimental values. The lens is assumed to be placed at the $z=0$ plane.

$=1: 4: 11$ ). Pulses last $100 \mathrm{~ns}$ (with no nitrogen) and $2-3$ $\mu s$ (with $\mathrm{N}_{2}$ ). The laser cavity is a half symmetric resonator, in which the distance between the curved mirror (radius $10 \mathrm{~m}$ ) and the planar (output) mirror is $103 \mathrm{~cm}$. Also, we have placed an intracavity diaphragm close to the curved mirror to attenuate the higher-order modes.

The experimental (squared) beam width along the transversal $x$-axis versus different distances in free space after the focusing lens is plotted in Fig. 2 for the value $d=10 \mathrm{~mm}$ of the diameter of the adjustable aperture inside the cavity. From these curves the respective beam quality parameter can be derived.

Fig. 3 shows the dependence of the beam quality parameter $Q$, given by Eq. (6), on both, the presence of nitrogen in the gas mixture and the size of the intracavity aperture. Note that the beam quality product $4 k^{2} Q$ for $d=8 \mathrm{~mm}$ is nearly unity with and without $\mathrm{N}_{2}$. From Fig. 3 we also see that the quality deteriorates for higher values of $d$, as expected (diffraction losses reduce and the number of laser modes would increase). Also the quality is worse when nitrogen is present in the gas mixture. This can be

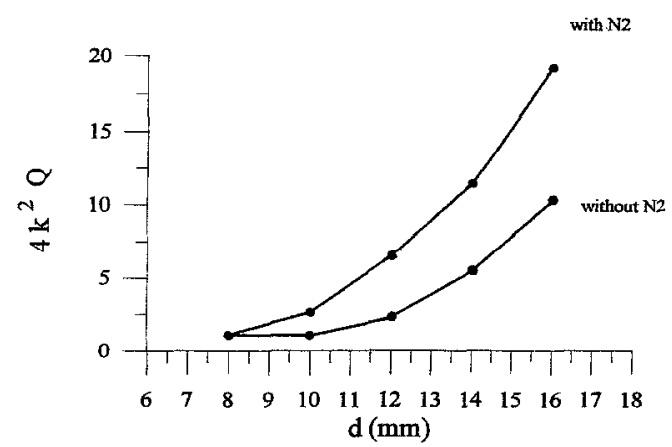

Fig. 3. Beam quality product $4 k^{2} Q$ for different values of the diameter of the intracavity diaphragm. understood by noting that longer pulses provide suplementary time to the higher-order modes for growing in amplitude after successive round trips (it should be remarked that it has been observed experimentally [9] that there is a delay of $\sim 300-400 \mathrm{~ns}$ between the moment the discharge begins and the moment when the laser pulse is strong enough to be detected by a linear photodetector over the noise level).

To prove the presence of multiple modes a well known sensitive test is to measure the so-called mode beats generated by heterodyne interference effects at different frequencies between the modes. The experimental set-up is sketched in Fig. 4: The pulsed beam is collected by a fast (1 ns rise time) photo-detector (Edinburgh PDM-2 photon drag) and registered by a digital oscilloscope (Tektronix DSA 602). A computer performs the Fourier transform of the laser pulse and provides the spectrum of the photodetector signal. Figs. 5 and 6 show several examples of mode beating for $d=10 \mathrm{~mm}$ with and without $\mathrm{N}_{2}$. The conclusions about the number of modes are summarized in Table 1.

Let us then consider the case in which the diameter of the cavity diaphragm is $d=10 \mathrm{~mm}$ and there is no nitro-

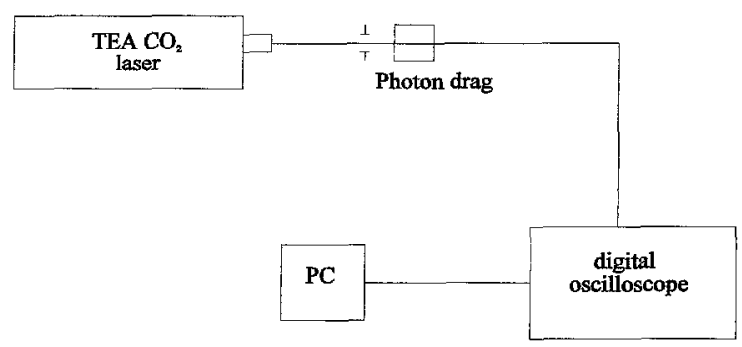

Fig. 4. Schematic of the experimental set-up for mode-beating measurements. 


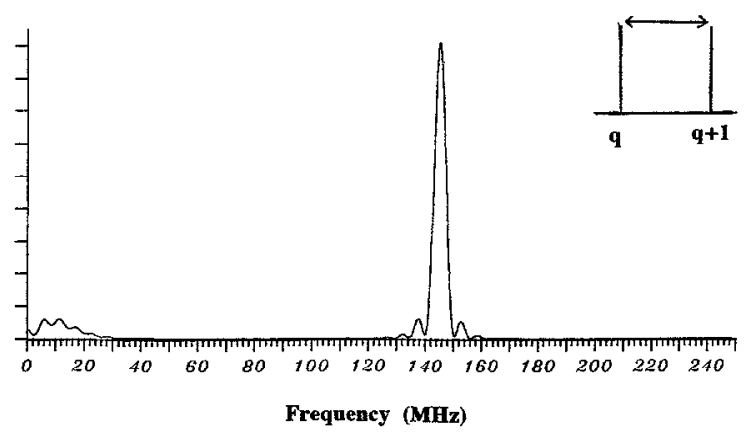

Fig. 5. Spectrum of the photodetcctor signal corresponding to mode beating for $d=10 \mathrm{~mm}$ with no nitrogen in the gas mixture. The peak frequency is associated with the beating between two adjacent axial mode frequencies (associated to the same spatial structure). Therefore, in this case there is only one mode.

gen in the gas mixture. In such a case, from the experimental data we get $\gamma=0.44 \mathrm{~mm}^{-1}, s=1.33$ and $r=0.26$. In addition, Fig. 5 shows that there is only one mode, $f_{a}$, whose beam quality product $4 k^{2} Q$ is nearly 1 , so that the spatial structure of this mode should be close to the pure Gaussian profile. Some difference, however, should exist between such mode and the Gaussian function $\varphi_{0}$, because $r$ differs from zero. Moreover, as was pointed out before, it should involve at least the second-order H-G function $\varphi_{2}(x)$ (cf. Eq. (28)). By taking this into account, we will consider that the pulse amplitude (as given by Eq. (12)) can be written in terms of the H-G functions $\varphi_{0}, \varphi_{1}$ and $\varphi_{2}$ in the form

$$
\begin{aligned}
f_{a}= & \gamma^{1 / 2}\left[A_{a 0}(\omega) \varphi_{0}(x)+A_{a 1}(\omega) \varphi_{1}(x)\right. \\
& \left.+A_{a 2}(\omega) \varphi_{2}(x)\right],
\end{aligned}
$$

where now (see Eqs. (18) and (19))

$$
\frac{1}{P_{a}} \int\left|A_{a p}\right|^{2} \mathrm{~d} \omega=R_{p p}^{(a)}
$$

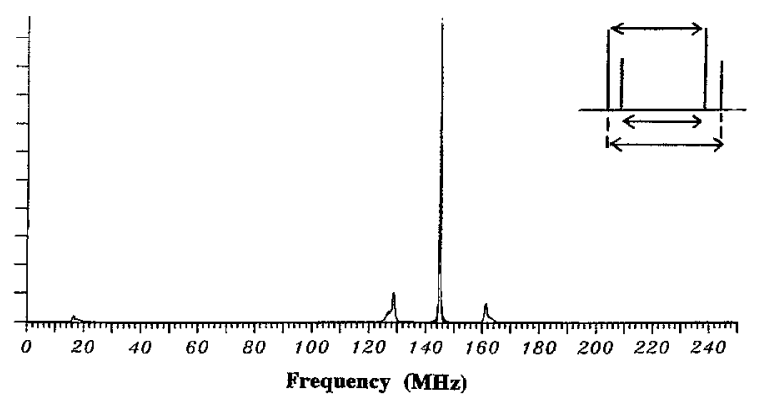

Fig. 6. The same as in Fig. 5 but now with the presence of nitrogen. The secondary peaks correspond to beating between transversal mode frequencies (associated to different spatial structures). Here we have two modes.
Table 1

\begin{tabular}{lll}
\hline & $d=8 \mathrm{~mm}$ & $d=10 \mathrm{~mm}$ \\
\hline $\begin{array}{l}\text { without } \mathrm{N}_{2} \\
\text { with } \mathrm{N}_{2}\end{array}$ & one mode & one mode \\
one mode & two modes \\
\hline
\end{tabular}

and

$P_{a}=\frac{1}{\gamma} \int\left|f_{a}\right|^{2} \mathrm{~d} x \mathrm{~d} \omega$.

The first-order H-G function, $\varphi_{1}(x)$, has been included in Eq. (31) because the diffraction losses associated to this term are likely to be lower than the losses of the higherorder H-G function $\varphi_{2}(x)$.

Note that integration of the pulse amplitude over the pulse length gives

$\int\left|f_{a}\right|^{2} \mathrm{~d} \omega \propto R_{00}^{(a)}\left|\varphi_{0}\right|^{2}+R_{11}^{(a)}\left|\varphi_{1}\right|^{2}+R_{22}^{(a)}\left|\varphi_{2}\right|^{2}$,

where the symbol $\propto$ denotes proportionality. This clarifics why the cocfficients $R_{p p}^{(a)}, p=0,1,2$, can be understood as the relative contribution of each (squared) $\mathrm{H}-\mathrm{G}$ function to the laser mode.

From Eqs. (27) and (28) it then follows

$1.07=R_{00}^{(a)}+3 R_{11}^{(a)}+5 R_{22}^{(a)}$,

$1=R_{00}^{(a)}+R_{11}^{(a)}+R_{22}^{(a)}$,

which implies

$R_{00}^{(a)}>0.948$,

$R_{11}^{(a)}<0.035$,

$R_{22}^{(a)}<0.017$.

In summary, we have that, in terms of percentages, the spatial structure would be composed of nearly $95 \%$ of the zero-order $\mathrm{H}-\mathrm{G}$ function, whereas contributions of the first- and second-order $\mathrm{H}-\mathrm{G}$ functions would be about $3.5 \%$ and $1.5 \%$, respectively. We thus see that, in this case, the cavity losses along with the presence of the active medium slightly distort the spatial structure of the beam, which would be purely Gaussian for the empty cavity.

If nitrogen is present in the gas mixture, we know from Table 1 that there are two modes. We will assume that one of them has the same spatial structure as the mode we have just analysed. Some physical reasons that justify this assumption are the following: In the measurements carried out on the $8 \mathrm{~mm}$ diaphragm, the values of $s$ and $r$ without $\mathrm{N}_{2}$ in the gas mixture were quite similar to those measured with the presence of $\mathrm{N}_{2}$. Given that, in both situations, only one mode exists (see Table 1), it seems reasonable to assume that in this case the greater length of the pulse (caused by the nitrogen) has no effect on the spatial structure of the lowest-order mode. Accordingly, when a diaphragm of larger diameter $(d=10 \mathrm{~mm})$ is used, diffrac- 


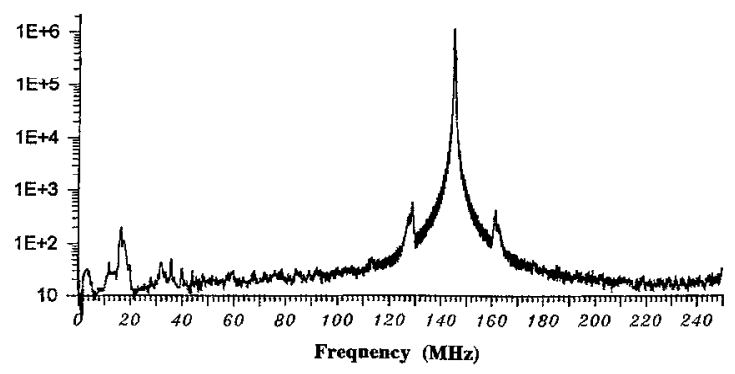

Fig. 7. The same as in Fig. 6 but now integrating throughout the spatial transversal section of the beam. Note that, in ordinates, we use logarithmic scale.

tion effects on the quasi-Gaussian lowest-order mode are likely to be smaller than in the previous case. Hence, the above referred mode would also maintain its structure when the pulse length increases. Taking this into account, we could consider in this case a global pulse amplitude, $\psi$, of the form

$\psi(x, \omega)=\alpha_{a} f_{a}(x, \omega)+\alpha_{b} f_{b}(x, \omega)$

where $f_{a}$ denotes the lowest-order mode that appears when no nitrogen is present (see Eq. (31)) and $f_{b}$ would represent the second-order mode structure. With regard to this mode, it should be noted that it is "almost" orthogonal to the fundamental mode, even though orthogonality in the strict sense is not completely fulfilled. To evaluate this fact we have observed the beating of such modes but now performing the spatial integration throughout the transversal section of the beam. The result is shown in Fig. 7. The secondary peaks correspond to the mode beating we are interested on (it involves $f_{a}$ and $f_{b}$ ), whereas the highest peak involves different frequencies but with the same spatial structure (complete orthogonality would imply no appearance of such secondary peaks). It should be mentioned that a similar effect could, however, result from the non-uniformity of the response of the detector. Here we assume that the main effect comes from slight non-orthogonality between modes. We will then write $f_{b}$ as follows:

$$
\begin{aligned}
f_{b}= & \gamma^{1 / 2}\left[A_{b 1}(\omega) \varphi_{1}(x)+A_{b 2}(\omega) \varphi_{2}(x)\right. \\
& \left.+A_{b 3}(\omega) \varphi_{3}(x)\right],
\end{aligned}
$$

where

$\frac{1}{P_{b}} \int\left|A_{b p}\right|^{2} \mathrm{~d} \omega=R_{p p}^{(b)}$

and

$P_{b}=\frac{1}{\gamma} \int\left|f_{b}\right|^{2} \mathrm{~d} x \mathrm{~d} \omega$.

In Eq. (41) $\varphi_{3}$ should be included by a similar argument to that used to introduce $\varphi_{2}$ in Eq. (31). The H-G function $\varphi_{2}$ has also been included because its diffraction losses are likely to be lower than the losses of $\varphi_{3}$. In addition, since $f_{a}$ and $f_{b}$ are associated with different sets of frequencies, it would follow that

$\int A_{a p}^{*}(\omega) A_{b p}(\omega) \mathrm{d} \omega=0$.

Let us now denote by $M$ the percentage of mode $f_{a}$ in the global pulse. In other words, we define

$M=\frac{\left|\alpha_{a}\right|^{2} P_{a}}{P}$,

where $P=\left|\alpha_{a}\right|^{2} P_{a}+\left|\alpha_{b}\right|^{2} P_{b}$. Then, if $R_{p p}$ represent the coefficients introduced in Eq. (18) for the global pulse $\psi$, we have

$R_{00}=M R_{00}^{(a)}$,

$R_{11}=M R_{11}^{(a)}+(1-M) R_{11}^{(b)}$,

$R_{22}=M R_{22}^{(a)}+(1-M) R_{22}^{(b)}$,

$R_{33}=(1-M) R_{33}^{(b)}$.

But in this case

$s-r=\sum_{p=0}^{3}(2 p+1) R_{p p}=1.71$,

along with

$1=\sum_{p=0}^{3} R_{p p}$.

Consequently we get

$M \geq 0.65$.

We thus have that, when nitrogen is present, the lowestorder mode structure constitutes, at least, $65 \%$ of the global pulse spatial shape.

\section{Conclusions}

By writing the pulse amplitude in terms of $\mathrm{H}-\mathrm{G}$ functions, a number of equations were found that enable us to link the spatial structure of the beam to its (measurable) second-order intensity moments integrated along the pulse length. We have also investigated from an experimental point of view the dependence of the beam quality parameter (integrated along the pulse length) of TEA $\mathrm{CO}_{2}$ laser pulses on the presence of nitrogen in the gas mixture, and also on the size of an adjustable diaphragm inside the laser resonator. It was found that the beam quality deteriorates for higher values of the diameter of the intracavity aperture, as well as when nitrogen is present in the gas mixture (longer pulses). For small enough values of such diaphragm, the beam quality product $4 k^{2} Q$ was shown to be nearly unity, independent of the presence of nitrogen. Finally, some of the above numerical values have been used to illustrate how to get more information about the 
spatial behaviour of a pulse: in particular, a number of consequences were inferred about the significance and the relative importance of each H-G term. It should be remarked that the formalism shown here applies for the bidimensional case. We plan to extend the results in subsequent papers to general tridimensional pulses.

\section{Acknowledgements}

The research work leading to this paper has been supported by the Comision Interministerial de Ciencia y Tecnología of Spain under Project TAP93-211, within the framework of EU-1269 project.

\section{References}

[1] S. Lavi, R. Prochaska, E. Keren, Appl. Optics 27 (1988) 3696

[2] M.J. Bastiaans, Optik 82 (1989) 173.

[3] A.E. Siegman, Proc. SPIE 1224 (1990) 2.

[4] J. Serna, R. Martínez-Herrero, P.M. Mejías, J. Opt. Soc. Am. A 8 (1991) 1096.

[5] H. Weber, Opt. Quantum Electron. 24 (1992) 1027.

[6] P.M. Mejías, R. Martínez-Herrero, Optics Lett. 20 (1995) 660.

[7] A.E. Siegman, Lasers (Oxford Univ. Press, Oxford, 1986) Chs. 16 and 19.

[8] R. Martínez-Herrero, P.M. Mejías, Optics Comm. 94 (1992) 197.

[9] F. Encinas, J.M. Guerra, IEEE J. Quantum Electron. 27 (1991) 891. 